\title{
Cluster of Differentiation
}

National Cancer Institute

\section{Source}

National Cancer Institute. Cluster of Differentiation. NCI Thesaurus. Code C78347.

Cell surface antigens that are differentially expressed on the surface of cells in both a tissue- and differentiation stage-specific manner. Identification of differentiation antigens can be used to characterize, and isolate, the types of cells in a sample and can identify cells that have undergone differentiation or transformation. 